\title{
ENSAYO SOBRE EL COVID-19 EN EL PERÚ: ALGUNAS REFLEXIONES EN MEDIO DE LA PANDEMIA $^{\circ 0}$
}

\author{
ESSAY ON COVID-19 IN PERU: SOME \\ REFLECTIONS IN THE MIDST OF THE PANDEMIC
}

Rafael Baldomero López Lozano*

enviado: 14 septiembre 2020 - aceptado: 22 octubre 2020

\section{Resumen}

El presente ensayo busca compartir algunas reflexiones en torno a la pandemia de COVID-19 en el Perú. La idea central refiere a que tanto el Estado como la sociedad peruana hicieron lo que pudieron en la medida de sus posibilidades y, a pesar de las cerca de 70000 muertes, es posible que se haya evitado la mayor catástrofe humanitaria de nuestra historia conocida. En primer lugar, si comparamos la epidemia de COVID-19 con otros eventos de nuestra historia. Por otro lado, hablando de las fortalezas y debilidades al momento de manejar la crisis. Y por último, una estimación de qué hubiera pasado de no haberse tomado ninguna medida.

Palabras clave: pandemia, Perú, historia, ciencias sociales.

Código JEL: N96.

\begin{abstract}
This essay seeks to share some reflections on the COVID-19 pandemic in Perú. The central idea refers to the fact that both the Peruvian State and society did what they could to the best of their ability and, despite the nearly 70,000 deaths, the

Las opiniones expresadas aquí son responsabilidad exclusiva del autor.

- López Lozano, R. B. (2021). Ensayo sobre el COVID-19 en el Perú: algunas reflexiones en medio de la pandemia. Estudios económicos, 38(76), 259-278

* Colegio de Michoacán A. C., México. Correo electrónico: lopezlozanorafael@gmail.com ORCID: https://orcid.org/0000-0002-0316-3695.
\end{abstract}


greatest humanitarian catastrophe in our known history may have been avoided. In the first place, if we compare the COVID-19 epidemic with other events in our history. By the other hand, we were talking about the strengths and weaknesses when handling the crisis. And finally, an estimate of what would have happened had no action been taken.

Keywords: pandemic, Peru, history, social sciences.

Code JEL: N96. 


\section{INTRODUCCIÓN}

Este ensayo busca compartir algunas reflexiones en torno a las repercusiones de la pandemia de COVID-19 en el Perú. Se parte de la pregunta de qué lugar podría tener este evento en nuestra sociedad si es que lo comparamos con otros acontecimientos en nuestra historia. Para responder esto, trato de tener una mirada de largo horizonte pero tomando en cuenta que este proceso implica vidas perdidas y familias que sufren por sus seres queridos.

Mi idea central es que se ha hecho todo lo posible, y probablemente ha sido mucho. Como hipótesis, probablemente esta pandemia ha sido el mayor reto que hemos tenido como peruanos en nuestra historia. Por otro lado, y tomando en cuenta nuestras carencias como sociedad, se evitó la mayor tragedia humana de nuestra historia conocida. Así, estuvimos muy cerca de tener un desastre humano de proporciones solamente vistas en la época de la conquista (Contreras, 2020). Ello se pudo evitar gracias a que el Estado y los ciudadanos hicieron lo que pudieron, a pesar de no tener una sociedad de bienestar.

Antes de empezar, sería necesario hablar un poco sobre el sistema de salud peruano. Este se encuentra compuesto por cuatro subsistemas. Primero están los seguros privados de clínicas y que son usados por la clase media/alta. Luego están los hospitales de los militares y policías. Después, tenemos la seguridad social (ESSALUD), usada por empleados con estabilidad laboral. Finalmente, tenemos el seguro integral de salud (SIS), el cual depende del Estado y está enfocado en los más pobres.

En el Perú, durante los últimos años hubo un incremento del presupuesto de salud. De acuerdo con el Ministerio de Economía y COMEX Perú, hemos visto que hemos pasado de 11000 a 17 millones en los últimos cinco años. Sin embargo, esta cantidad se puede considerar poca para satisfacer las necesidades de 30 millones de personas.

A nivel regional también nos encontramos en desventaja. De acuerdo con el Banco Mundial, durante 2016 teníamos un gasto menor en proporción con el promedio latinoamericano (4\%) y otros países miembros de la Alianza del Pacífico: Chile (5\%) y Colombia (3.7). Si comparamos esto con países de la OCDE, la diferencia resulta abismal porque destinan en promedio un $10 \%$ de su PBI en salud.

Los datos del personal destinado a salud también evidencian ciertas debilidades. De acuerdo con estimaciones del Ministerio de Salud y la Cooperación 
Italiana, teníamos cerca de 13 médicos por cada 10000 habitantes. Esto resulta ser menos de la mitad que en los países miembros de la OCDE (33 por cada 10000 ).

Gráfico 1. Comparación del gasto público en salud desde 2013 a 2018 (en millones de soles)

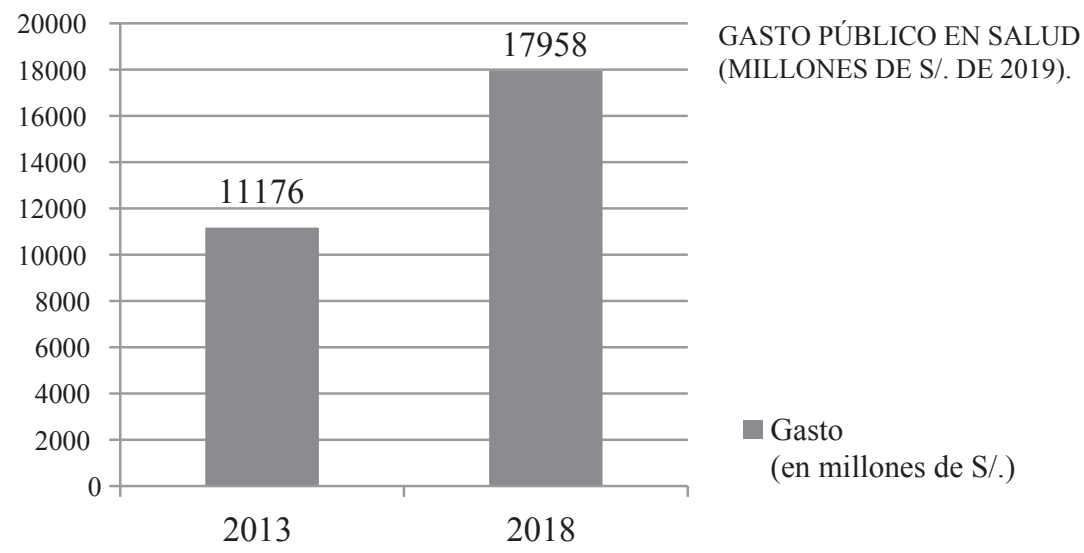

Fuente: Gestión, 2019.

Gráficos 2. Comparación de gasto peruano en salud GASTO DEL GOBIERNO EN SALUD (\% PBI 2016)

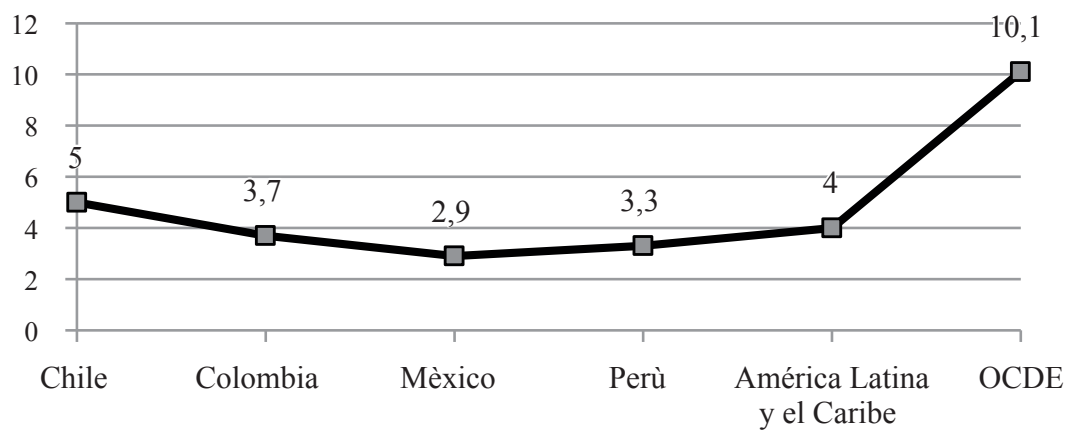

Fuente: Gestión, 2019. 
Con una breve vista de estos datos, es posible apreciar la debilidad del sistema de salud peruano. En ese sentido, podríamos decir que el país no se encontraba preparado para enfrentar una pandemia. Esto porque, desde antes, nuestro sistema estaba siempre al borde del colapso.

Debido a esto, mis argumentos se centran en tres puntos. Primero, en la comparación de la epidemia con otros eventos catastróficos dentro de la historia peruana conocida. Después, en la mención de las carencias y fortalezas al momento de afrontar la emergencia. Y, por último, se propone analizar qué hubiera sucedido en el caso de no haber hecho nada. Creo que estos argumentos pueden ayudarle al lector a conocer mi posición.

\section{EL LUGAR DEL COVID-19 EN LA HISTORIA PERUANA}

Si vemos la cantidad de víctimas cobradas por la epidemia en la historia peruana, el COVID-19 demoró un año en matar más gente que la época del terrorismo (1980-2000) ${ }^{1}$. Esto se puede apreciar por la cantidad de muertos y peor si sacamos el promedio de víctimas anuales. Frente a ello, podría argumentarse que faltaría contextualizar pero esto nos facilita a conocer la magnitud del problema.

En comparación, eventos como la Guerra del Pacífico o el terrorismo han tenido una menor cantidad de víctimas. Esto ubica ya a la epidemia de COVID-19 como una de las 10 principales catástrofes conocidas en nuestra historia. Es decir, en casi 6 meses la epidemia del COVID-19 se ubica en el puesto 5.

En esta escala, encontramos que la conquista se cobra la muerte de más de 7 millones de personas. Después, ubicamos el genocidio del Putumayo con aproximadamente 250000 personas fallecidas, mayormente indígenas. Luego, la rebelión de Túpac Amaru II y el terremoto de Ancash de 1970 se cobraron la vida de 100000 personas.

Seguido a esto, encontramos la epidemia del COVID-19 con un promedio de 70000 personas fallecidas, superando la época del terrorismo en el Perú (69 000 personas) y a todas las guerras civiles del primer caudillismo (1829-1859). Al final, se encuentra la Guerra del Pacífico y la Independencia con 20000 fallecidos.

1 Comisión de la Verdad y Reconciliación, 2004. 
De acuerdo a diversas estimaciones, los eventos históricos que cobraron la mayor cantidad de víctimas afectaron más a los pueblos originarios (Contreras, 2020) (Barclay \& Santos, 2002) (CVR, 2004). Podemos ver entonces que nuestra sociedad, tan profundamente clasista y racista, nos hace ignorar la mayoría de estos hechos. Es decir, son períodos donde se eliminó sistemáticamente a la población más desfavorecida.

Tabla 1. Diez principales catástrofes conocidas en la historia del Perú (desde 1532) por número de muertos

\begin{tabular}{ll}
\hline Evento histórico & Total de víctimas mortales \\
\hline Conquista del Perú (1533-1572) & $7700000^{1}$ \\
\hline Genocidio del Putumayo (1879-1912) & $250000^{2}$ \\
\hline Rebelión de Túpac Amaru II (1780-1781) & $101000^{3}$ \\
\hline Terremoto de Ancash de 1970 (1970) & $100000^{4}$ \\
\hline Epidemia de COVID-19 de 2020 & $71000^{5}$ \\
\hline Época del terrorismo en el Perú (1980-2000) & $69000^{6}$ \\
\hline Guerras Civiles del Caudillismo Militar (1829-1859) & $41000^{7}$ \\
\hline Guerra del Pacífico (1879-1883) & $20000^{8}$ \\
\hline Guerras de Independencia (1811-1826) & $20000^{9}$ \\
\hline
\end{tabular}

Fuente: elaboración propia.

La magnitud de otros eventos puede verse en el siguiente gráfico (gráfico 1). A primera vista, la conquista es por lejos el evento que más cantidad de víctimas tuvo. Sin embargo, lo importante aquí es observar que la epidemia de COVID-19 del 2020 se encuentra en el puesto 5 de nuestra historia.

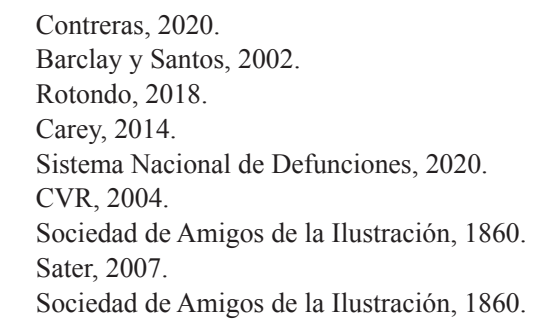


Por otro lado, cuando vemos el número promedio de muertos, la cosa se pone peor. Aquí, la epidemia del COVID-19 pasa del quinto puesto al tercer lugar. Ello nos debe dar una muestra de la gravedad del problema.

Gráfico 3. Total de víctimas mortales de distintos eventos históricos

Total de víctimas mortales

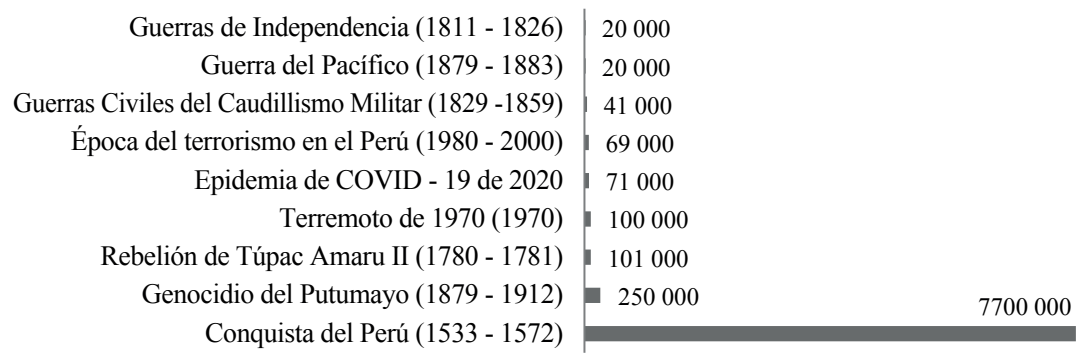

Fuente: elaboración propia.

Tabla 2. Diez principales catástrofes conocidas en la historia del Perú (desde 1532) en muertos por año

\begin{tabular}{ll}
\hline Evento histórico & Número de muertos por año \\
\hline Conquista del Perú (1533-1572) & 192500 \\
\hline Terremoto de 1970 (1970) & 100000 \\
\hline Epidemia de COVID-19 de 2020 & 71000 \\
\hline Rebelión de Túpac Amaru II (1780-1781) & 50500 \\
\hline Genocidio del Putumayo (1879-1912) & 7576 \\
\hline Guerra del Pacífico (1879-1883) & 5000 \\
\hline Época del terrorismo en el Perú (1980-2000) & 3450 \\
\hline Guerras Civiles del Caudillismo Militar (1829-1859) & 1367 \\
\hline Guerras de Independencia (1811-1826) & 1333 \\
\hline
\end{tabular}

Fuente: elaboración propia. 
De acuerdo con la tabla 2, la Conquista del Perú tiene la mayor cantidad de muertos por año, casi 200000 personas. Después está el terremoto de Ancash de 1970 con cerca de 100000 víctimas. Luego de estas dos grandes tragedias ya encontramos al COVID19 con cerca de 70000 personas fallecidas.

En cuarto lugar, está la rebelión de Túpac Amaru con 50000 personas fallecidas por año. Seguido a ello, el genocidio amazónico mató a 7000 personas al año. La época del terrorismo y la Guerra del Pacífico están después, con 5000 y 3500 muertes, respectivamente. Al final, se encuentra la cantidad de fallecidos en el caudillismo militar y la Independencia con menos de 1500 muertes promedio.

Con estos indicadores apreciamos la magnitud del problema. Es decir, durante menos de un año la sociedad peruana ha tenido que afrontar un evento pocas veces visto en términos de letalidad a lo largo de nuestra historia conocida. Solamente teniendo una perspectiva histórica, vemos que la epidemia del COVID19 resulta ser algo muy serio.

Gráfico 4. Cantidad de víctimas mortales en cada año que duró el proceso histórico

Número de muertos por año

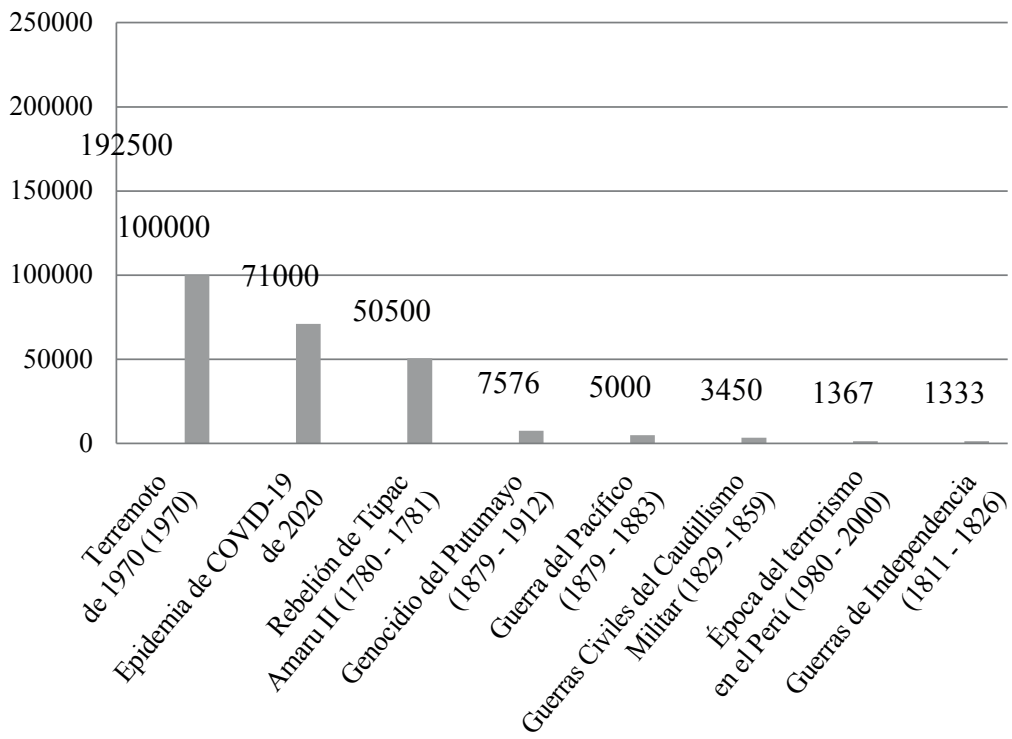

Fuente: elaboración propia. 
Tomando en cuenta el gráfico 2, el COVID19 es uno de los eventos históricos más letales en la historia de nuestro país. Cuando nos referimos al número de víctimas promedio, pasa del quinto al tercer lugar dentro del ranking. Entonces, sería bueno preguntarnos: ¿qué consecuencias va a tener a largo plazo si cosas mucho menos letales alteraron profundamente la sociedad peruana?

\section{NUESTRAS DEBILIDADES Y FORTALEZAS AL MOMENTO DE AFRONTAR LA CRISIS}

Siguiendo con este ensayo, quiero analizar nuestras carencias y fortalezas al momento de afrontar la epidemia. Esto lo quiero realizar de la forma más amplia posible, como científico social y como una persona que ha vivido un acontecimiento que para muchos es doloroso. Por lo tanto, soy consciente de que mi posición guarda subjetividades, pero quiero tratar de hacer el ejercicio de un científico social que analiza un determinado fenómeno.

En ese sentido, la razón principal que explicaría la cantidad de muertos es la ausencia de un Estado de bienestar. Durante el período de crecimiento económico por la bonanza de los minerales, se dejó de lado la implementación de un sistema de bienestar social capaz de brindar servicios públicos de calidad a toda la población. Los principales servicios públicos que faltan serían: la ausencia de pensiones para todos los sectores sociales, seguros de desempleo, vivienda social, educación pública con infraestructura adecuada y, sobre todo, un sistema de salud (Béjar, 2012).

Por otro lado, un sector abandonado durante esta época fue la investigación académica. Esto podría explicarse bajo la siguiente idea: no somos un país desarrollado y primero necesitamos estabilidad macroeconómica, no debemos gastar dinero en cosas tan "poco urgentes". Sin embargo, el COVID19 demostró que estos servicios, lejos de ser gastos, son lo básico para toda sociedad civilizada. Para ello, cabría preguntarnos: ¿habría sido necesaria una cuarentena que perjudicó toda la actividad económica si hubiéramos tenido un sistema de salud fuerte?

De esta forma, hemos llegado a esta cantidad de muertos por los prejuicios existentes dentro de los diseñadores de política. Esto motivó que exista un sistema de salud con carencias, que se tuvo que ir construyendo en el camino. Además, se pensaba que no era necesaria la inversión en fortalecer los servicios del Estado puesto que poco a poco se iba cubriendo por el sector privado. 
Este error también se veía acompañado por la necesidad de "gastar poco" en confluencia con intereses privados que mantenían abandonado el sector. Por un lado, los políticos asumían que promover la inversión privada era cobrar la menor cantidad de impuestos, lo que favorecía a las grandes empresas. De manera que la ausencia de un Estado de bienestar capaz de enfrentar la epidemia tiene como responsables la dejadez por realizar una reforma tributaria que le permita al Estado tener los recursos reales para implementar los servicios que siempre debió tener.

Sin embargo, creo que hay fortalezas igual de destacables, cosas que ni siquiera pudiéramos haber imaginado hasta hace unos 20 años (Quiroz, 2013). Durante los últimos años, se fortaleció el sistema democrático, en el sentido de que poco a poco se fueron realizando elecciones regulares. Eso brindó cierta estabilidad, necesaria para que el Estado pudiera dedicarse a su labor en el sentido de implementar políticas. Poco a poco la democracia como sistema político de alternativas de gobierno se acentuó en el país.

Con la profundización de un Estado democrático, cada institución pública ha tenido mayor independencia para cumplir sus funciones. Ello llevó a la existencia de especialistas capaces, formándose dos dimensiones: policy y politics. Podríamos decir que no tenemos suficiente institucionalidad, aunque definitivamente tenemos más que hace 40 años (Cotler, 1978).

Evidencia de ello es que en los últimos años hemos podido apreciar una mayor división entre los poderes del Estado. Esto implica que ningún grupo es capaz de acaparar todas las instituciones y nuestro sistema de justicia se ha hecho más independiente del poder. Prueba de ello es que hayamos sido capaces de juzgar e investigar a un expresidente por lesa humanidad, y a otros por corrupción dentro del marco de la ley. Así, creo necesario ver estos avances, que no son poca cosa, mucho más si tomamos en cuenta cómo era el país hace un siglo (Burga \& Flores, 1984).

Otra cosa para nada superficial es que somos una nación. Esto porque, independientemente de nuestros conflictos de clase y nuestro racismo, la epidemia del COVID19 ha sido tratada como un problema nacional. A lo largo de nuestra historia, en períodos de emergencia como estos, siempre pudimos apreciar que el quiebre del Estado termina en que cada grupo social se salva como puede (Quiroz, 2013). En este caso, el país conserva un gobierno, que rinde cuentas, ha podido brindar información aceptable y en todos los sectores sociales se mantuvo la idea de que esto era un problema colectivo. 
Es verdad, existen fracturas en la sociedad peruana que tienen que ver con conflictos de clase y racismo presentes desde siempre (Cotler, 1978) (CVR, 2004). También es real que muchos grupos privilegian sus intereses más inmediatos. Sin embargo quiero hacer las siguientes preguntas: ¿qué sector ha dicho que la cuarentena es innecesaria? ¿Quién ha protestado por el uso de mascarillas? ¿En los peores momentos de la emergencia se han visto manifestaciones o lobbies desacatando las disposiciones del gobierno adrede?

Además, nuestro sistema de salud se movió lentamente, pero lo hace. Prueba de ello son la cantidad de pruebas realizadas, las personas recuperadas y médicos que aprendieron rápido de la enfermedad y en cada momento realizan mejoras en los tratamientos. Nuestro sistema de salud, estando siempre al borde del abismo, nunca llegó a colapsar totalmente. En ese sentido, quiero afirmar que todo el país ha intentado contener la propagación de la enfermedad en la medida de lo posible. Esto tomando como referencia las políticas implementadas por el Estado (Huamaní, 2020). Así, la dificultad fue más en términos materiales que de consciencia.

Estas medidas implementadas por el gobierno, si bien tomaron como referencia a los países centrales, fueron adaptadas a la realidad social del país. La primera de ellas que ayudó a frenar los contagios fue la suspensión de eventos sociales y cierre de fronteras, lo cual evitó la aglomeración de personas. Por otro lado, tenemos el uso obligatorio de mascarillas para que las pocas aglomeraciones que había no empeoraran la situación. Finalmente, la concentración de todas las instituciones públicas así como movilizar a toda la sociedad en hallar la forma de enfrentar a la epidemia. De manera que es posible destacar dos puntos importantes: la decisión política de enfrentar la epidemia y la puesta en marcha de mecanismos de solidad por parte de la ciudadanía.

En ese sentido, podemos decir que la mayoría de la población trató de acatar y respetar el estado de emergencia. La única razón por la que no se cumplió la cuarentena más estricta ha sido por necesidad económica, no por desidia de las personas. Esto fue posible porque se implementaron mecanismos de solidaridad entre la población en paralelo al mantenimiento del orden a nivel estatal.

Por ejemplo, se puede ver que hay medidas que se acatan a pesar de la poca presencia numérica de fuerzas militares y policiales en las calles. Una de ellas es el toque de queda en las noches y la otra el uso de mascarillas. Eso quiere decir que los peruanos han tratado de hacer todo lo posible por enfrentar la epidemia, siendo conscientes que puede amenazar sus vidas. 


\section{III. ¿QUÉ HABRÍA PASADO SI NO HACÍAMOS NADA?}

Si bien no pasó y se hizo la cuarentena, es válido pensarlo. Por este motivo, creo que tratar de aproximarnos a una respuesta puede ser útil.

Para ello, propongo realizar el cálculo. Este tiene que ver con la cantidad de muertos de no haberse realizado ninguna medida para contener el contagio. Si bien la cuarentena se realizó y se prolongó por cerca de tres meses, deberíamos siquiera pensar en qué hubiera pasado si no se hubieran tomado este tipo de medidas. Sería bueno preguntarnos: ¿qué hubiera pasado se dejábamos que el COVID-19 se propagara sin ningún control por todo el país?

En ese sentido, quiero citar el cálculo realizado por Pinedo y Pérez (2020), una estimación de la cantidad de infectados por COVID-19. Este arroja unas cifras que pueden resultar muy aterradoras. Si bien no menciona explícitamente las víctimas, es posible tener un aproximado del número de muertos.

Si tomamos en cuenta que teníamos 250 camas UCI al inicio de la pandemia, las personas que necesitarían esas camas y no tuvieran acceso morirían inevitablemente. En ese sentido, se puede calcular que probablemente hubieran fallecido entre los meses de abril y julio cerca de 1828091 personas por la epidemia de COVID-19 en el Perú. Este número se explica tomando en cuenta modelos matemáticos usados para predecir la expansión de una enfermedad tan contagiosa.

Podría pensarse que es exagerado, pero este tipo de cálculos no serían del todo irreales. Esto sí podría pasar si teníamos frente a nosotros una pandemia similar a la gripe española de 1918 y si tomamos en cuenta que no teníamos un sistema de salud preparado para afrontarla. Así, no es irracional suponer que no hacer nada hubiera significado la muerte de cerca de dos millones de personas.

De acuerdo con estos datos, la velocidad de contagio se produce con tal rapidez que el número se vuelve totalmente inmanejable a los pocos días. Así, los peores meses hubieran sido entre abril y mayo, con un promedio de 100000 personas fallecidas diarias por necesitar una cama UCI y no encontrarla. La dinámica de la epidemia producía un pico de muertes y esta iba descendiendo conforme pasaba el tiempo.

En el gráfico 3, vemos esto con más claridad. Desde el 15 de abril se hubiera visto una gran cantidad de muertes, las cuales hubieran crecido a ritmos muy altos dos semanas después. De ahí, hubiéramos llegado a un pico durante el mes de mayo y tendríamos una caída constante hasta el mes de julio. 
Tabla 3: Estimación de las personas fallecidas por COVID19 si no se hubiera tomado ninguna medida

\begin{tabular}{|c|c|}
\hline Fecha & Muertos \\
\hline $15-\mathrm{abr}$ & 4476 \\
\hline $30-a b r$ & 122654 \\
\hline 01-may & 136490 \\
\hline 02-may & 148472 \\
\hline 03-may & 158008 \\
\hline 04-may & 164702 \\
\hline 05-may & 168362 \\
\hline 06-may & 168990 \\
\hline 07-may & 166796 \\
\hline 08-may & 162242 \\
\hline 09-may & 155856 \\
\hline 10-may & 148116 \\
\hline 15-may & 102270 \\
\hline 01-jun & 16648 \\
\hline 15-jun & 3076 \\
\hline 30-jun & 321 \\
\hline 01-jul & 258 \\
\hline 02-jul & 202 \\
\hline 03-jul & 152 \\
\hline Total & 1828091 \\
\hline
\end{tabular}

Fuente: Pine do y Pérez (2020).

De esta forma, el control de la epidemia resultaba ser absolutamente necesario para asegurar la vida de millones de personas. De no haberlo hecho, la cantidad de víctimas fallecidas por la epidemia sería simplemente inimaginable. Frente a ello, no había discusión entre salvar la economía o la salud de la gente, ¿de qué economía hablamos si tenemos cerca de dos millones de muertos? 
Gráfico 5. Evolución de la cifra de fallecidos por COVID-19 de no tomarse ninguna medida

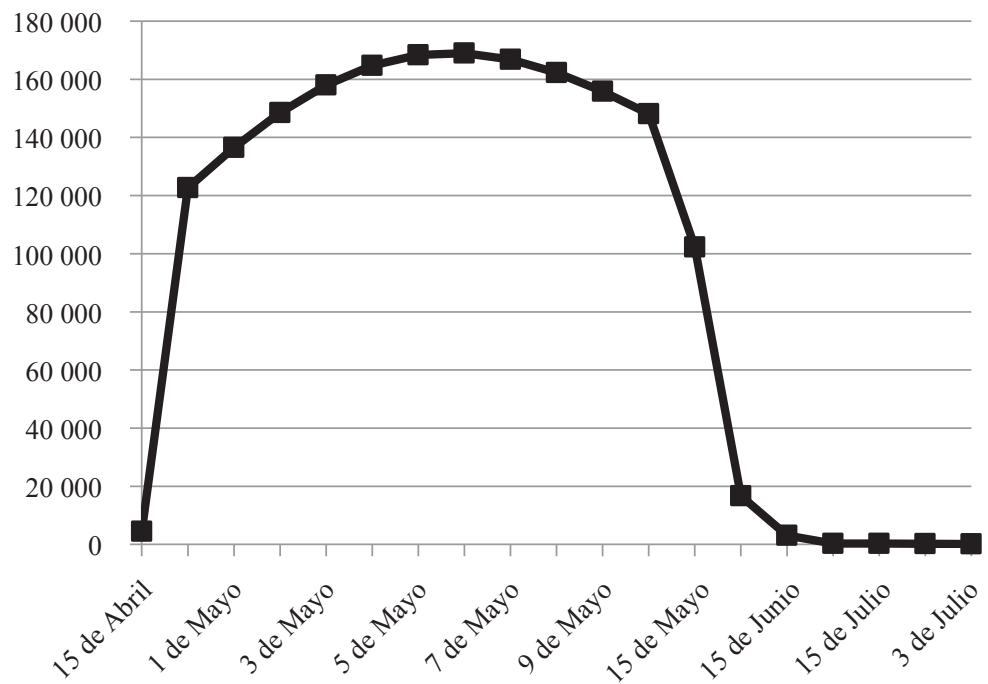

Fuente: Pinedo y Pérez (2020).

Con esta estimación, las cosas cambian completamente si comparamos la epidemia con otros eventos en la historia peruana. Si clasificamos el número de víctimas totales por una enfermedad no controlada, solamente la conquista hubiera tenido una mayor cantidad de víctimas. Así, la propagación de la enfermedad habría significado sin duda una tragedia humanitaria de dimensiones mucho mayores que la actual.

De acuerdo con esta nueva estimación, la conquista se mantendría primera con cerca de 7 millones de víctimas. Después, tendríamos la epidemia de COVID-19 con casi dos millones de personas. Finalmente, los otros eventos mantendrían su posición.

Es decir, si no se hubieran tomado medidas para contener los contagios, hubiéramos tenido más fallecidos en un año que en todas las guerras que libró el país a lo largo de su historia. Ello solamente implica un recuento de los fallecidos, mas no las consecuencias sociales, económicas y políticas de una tragedia de tal magnitud. En ese sentido, podríamos preguntarnos: ¿qué habría pasado con el país considerando que eventos menos letales alteraron las estructuras sociales de forma drástica? 
Tabla 4. Diez principales catástrofes conocidas en la historia del Perú (si no se hubiera hecho nada por la pandemia)

\section{Evento histórico}

Conquista del Perú (1533-1572)

Epidemia de COVID-19 de 2020

Genocidio del Putumayo (1879-1912)

Rebelión de Túpac Amaru II (1780-1781)

Terremoto de 1970 (1970)

Época del terrorismo en el Perú (1980-2000)

Guerras Civiles del Caudillismo Militar (1829-1859)

Guerra del Pacífico (1879-1883)

Guerras de Independencia (1811-1826)
Total de víctimas mortales

7700000

1828091

250000

101000

100000

69000

41000

20000

20000

Fuente: elaboración propia.

El gráfico (gráfico 4) nos ayuda a comprender la dimensión de la tragedia. En este se puede apreciar que las epidemias no controladas cobran una cantidad de víctimas mortales abrumadoramente superior a guerras o genocidios. Comparativamente, la época del terrorismo y todas las guerras libradas por el Perú no llegarían a pasar la cantidad de muertos si no se controlaba la epidemia del COVID19.

El escenario solo empeora si sacamos la cantidad de muertos por año. En este caso, la epidemia de COVID19 hubiera sido diez veces peor que la conquista. De acuerdo con lo visto, a la conquista le tomó 40 años matar cerca de 7 millones de personas, mientras que a la epidemia de COVID19 le hubiera tomado menos de un año acabar con la vida de cerca de 2 millones de personas.

Tomando en cuenta estos datos, la epidemia hubiera sido el evento más letal de nuestra historia conocida de acuerdo con el número de muertos por año, con cerca de 2 millones de personas. Después, se habría ubicado la Conquista del Perú con cerca de 200000 personas al año. Luego tendríamos las víctimas del terremoto de Ancash de 1970 con un aproximado de 100000 personas fallecidas. 

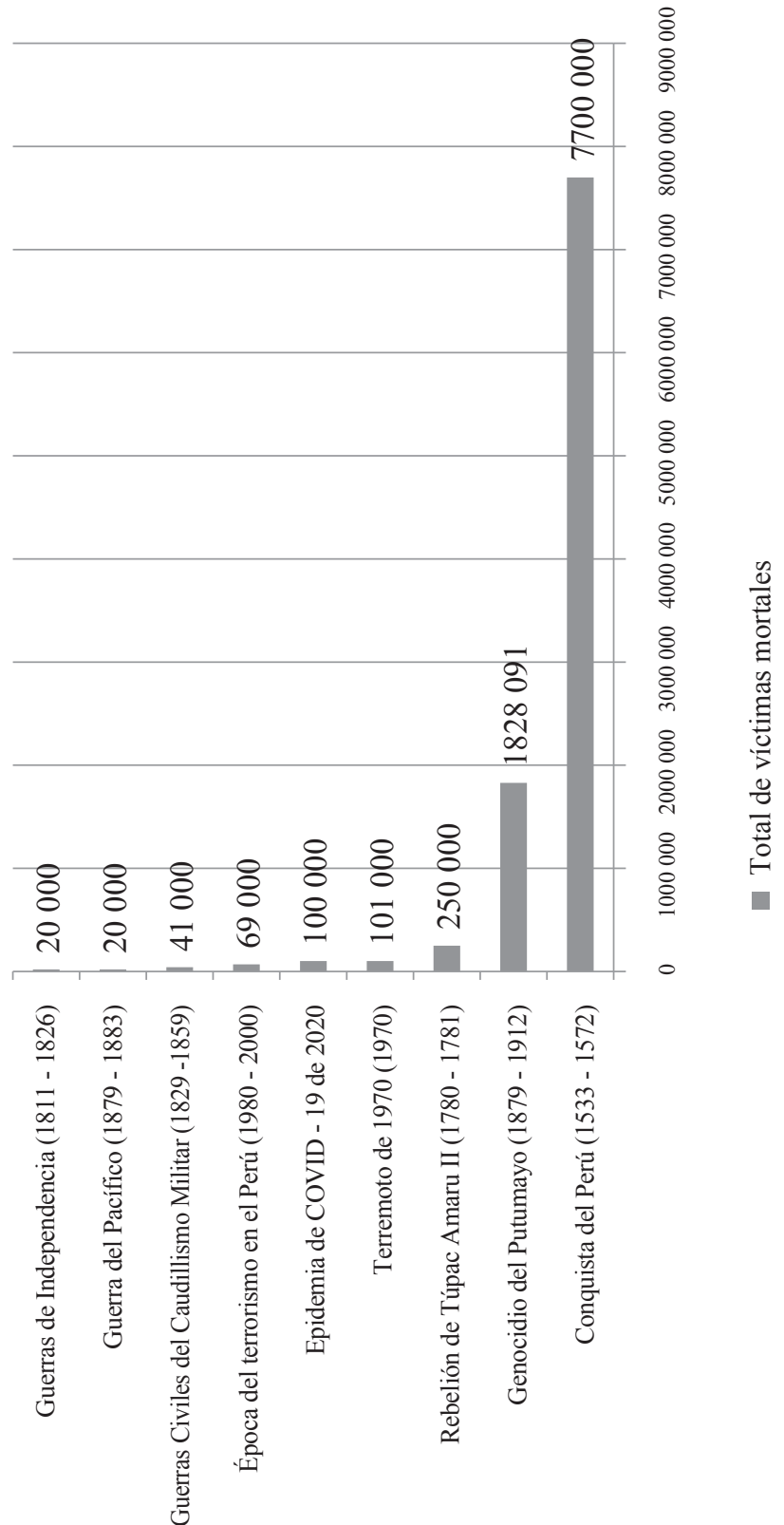
Seguido a esto, encontraríamos la Rebelión de Túpac Amaru II con cerca de 50000 personas fallecidas en dos años. Después, encontramos al genocidio del Putumayo (7 000), la Guerra del Pacífico (5 000) y la época del terrorismo (3 500). Finalmente, las guerras de la independencia y el caudillismo militar tendrían cerca de 1300 víctimas cada uno.

Creo que esto nos permite apreciar un escenario probable en caso de que no se hubiera tomado ninguna medida por parte del Estado y la sociedad. En este caso hipotético, nos encontraríamos frente a un panorama que ni siquiera podríamos imaginarnos. Este sería el siguiente: 1.8 millones de fallecidos en menos de un año, con una economía totalmente destrozada y sin saber qué depararía en el futuro.

Gráfico 7. Promedio de fallecidos por año en cada catástrofe histórica (de no haberse tomada ninguna medida contra el COVID-19)

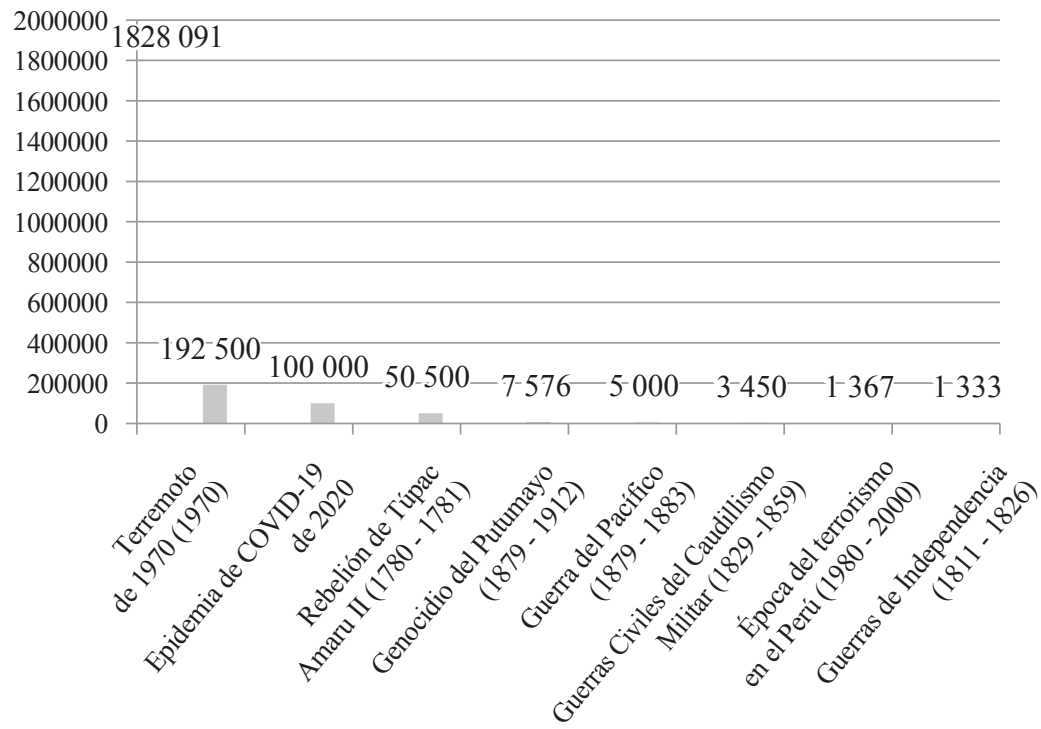

Fuente: elaboración propia.

\section{CONCLUSIONES}

A modo de conclusión, el balance general de la epidemia del COVID19 en el Perú podría ser el siguiente: tanto el Estado como la sociedad peruana hicieron lo que pudieron en la medida de sus posibilidades y, a pesar de las cerca de 70000 muertes, es posible que se haya evitado la mayor catástrofe humanitaria de nuestra historia conocida. 
En primer lugar, porque, potencialmente, la epidemia del COVID19 ha hecho en menos de un año lo que guerras enteras demoraron cerca de 5 o 20 años. $\mathrm{Al}$ momento de ver las cifras de muertos por años y el conteo general de las principales catástrofes demográficas en nuestra historia conocida, ello resulta evidente. Así, muy pocos eventos en nuestra historia son comparables a la cantidad de víctimas dejadas por la epidemia de COVID-19.

Por otro lado, a pesar de no contar con un Estado de bienestar capaz de brindar un sistema de salud eficiente a los ciudadanos, este ha podido poco a poco adaptarse a la emergencia y proponer soluciones. Prueba de ello es la cantidad de recuperados de la enfermedad, que todos los ciudadanos hayan asumido la responsabilidad de llevar mascarillas y salir lo menos posible. Si tomamos en cuenta que un grupo hizo poco caso a la cuarentena, esto se debió principalmente a la necesidad económica.

Finalmente porque, tomando en cuenta la propagación de la enfermedad, la cantidad de muertos si no se hubiera hecho nada hubiera sido inimaginable. Si esto no hubiera ocurrido, probablemente estaríamos frente a una tragedia infinitamente superior. En ese sentido, si solamente hubiera sido el doble, habríamos tenido 140000 familias enlutadas en un año.

Evidentemente, es muy pronto para conocer las consecuencias pero creo que estos deberían ser las principales metas. Estas deben girar en torno a asegurar nuestro desarrollo como país y, considero, son nuestros principales desafíos.

En primer lugar, la implementación de un Estado de bienestar capaz de asegurar el cumplimiento de los derechos sociales de todos los ciudadanos. Por otro lado, apostar por un sistema académico potente y capaz de producir sus propios conocimientos, donde el principal activo de los países sea el manejo de la información. Y, finalmente, ser conscientes del nuevo contexto internacional producto de haber vivido la pandemia más fuerte de los últimos cien años.

\section{REFERENCIAS BIBLIOGRÁFICAS}

Corporación Turca de Radio y Televisión en Español. (19 de junio de 2018). Perú tiene 12,8 médicos por cada 10000 habitantes, muy debajo de paises OCDE. (Sección Vida y Salud). Recuperado de https://www.trt.net.tr/espanol/viday-salud/2018/06/19/peru-tiene-12-8-medicos-por-cada-10-000-habitantesmuy-abajo-de-paises-ocde- 995479 
Barclay, F., \& Santos, F. (2002). La frontera domesticada: historia económica y social de Loreto, 1850-2000. Lima: Fondo Editorial PUCP.

Béjar, H. (2012). Mito y utopía: relato alternativo del origen republicano del Perú. Lima: Achebe Ediciones.

Burga, M. y Flores, M. (1984). Apogeo y crisis de la República Aristocrática. Lima: Ediciones Rikchay Perú.

Carey, M. (2014). Glaciares, cambio climático y desastres naturales - Ciencia y sociedad en el Perú. Lima: Instituto Francés de Estudios Andinos - IFEA, Instituto de Estudios Peruanos - IEP.

Comisión de la Verdad y Reconciliación. (2004). Hatun Willakuy: Versión abreviada del Informe Final de la Comisión de la Verdad y Reconciliación. Recuperado de https://lum.cultura.pe/cdi/sites/default/files/documento/pdf/ hatun_willakuy_0.pdf

Contreras, C. (2020). La crisis demográfica del siglo XVI en los Andes: una discusión acerca de sus dimensiones y consecuencias. Diálogo andino, (61), 7-25. Recuperado de https://dx.doi.org/10.4067/S0719-26812020000100007

Cotler, J. (1978). Clases, Estado y Nación en el Perú. Lima: Instituto de Estudios Peruanos.

García, E. (26 de mayo de 2019). Perú gasta en salud por debajo del promedio de América Latina. Gestión. Recuperado de https:/gestion.pe/economia/ comex-peru-gasta-salud-debajo-promedio-america-latina-268172-noticia/

Huamaní, C., Timaná-Ruiz, R., Pinedo, J., Pérez, J., \& Vásquez, L. (2020). Condiciones estimadas para controlar la pandemia de COVID-19 en escenarios de pre y poscuarentena en Perú. Rev Peru Med Exp Salud Pública (2), 37.

Huamaní, C., Timaná-Ruiz, R., Pinedo, J., Pérez, J., \& Vásquez, L. (2020). Condiciones estimadas para controlar la pandemia de COVID-19 en escenarios de pre y poscuarentena en el Perú. Revista Peruana de Medicina Experimental y Salud Publica, 37(2), 195-202. Recuperado de https://dx.doi. org/10.17843/rpmesp.2020.372.5405

Pinedo Taquia, J., \& Pérez Núñez, J. (2020). Estimación de la propagación del coronavirus 2019 (COVID-19) en Perú usando el modelo SIR. Lima: Ministerio de Salud del Perú.

Quiroz, A. (2013). Historia de la corrupción en el Perú. Lima: Instituto de Estudios Peruanos.

Rotondo, A. (2018). La Oración de la tarde: novela escrita sobre el célebre drama del mismo título original de Luis Mariano de Larra. Madrid: Imprenta y Litografía Militar del Atlas.

Saer, W. (2007). Andean Tragedy: Fighting the War of the Pacific, 1879-1884. Lincoln and London: University of Nebraska Press. 
Sociedad de Amigos de la Ilustración (1860). Revista del Pacífico. Literaria y Científica. Tomo II. Valparaíso: Imprenta y librería del Mercurio de Santos Tornero.

(C) 2021 por los autores; licencia no exclusiva otorgada a la revista Estudios económicos. Este artículo es de acceso abierto y distribuido bajo los términos y condiciones de una licencia Atribución-No Comercial 4.0 Internacional (CC BY-NC 4.0) de Creative Commons. Para ver una copia de esta licencia, visite http://creativecommons.org/licenses/by-nc/4.0 